Check for updates

Cite this: RSC Adv., 2019, 9, 11433

\title{
Contact lenses for continuous corneal temperature monitoring†
}

\author{
Rosalia Moreddu, (D) *ab Mohamed Elsherif, ${ }^{\mathrm{b}}$ Haider Butt, (D) ${ }^{\mathrm{c}}$ Daniele Vigolo (D) ${ }^{\mathrm{b}}$ \\ and Ali K. Yetisen ${ }^{a}$
}

Temperature variation is a ubiquitous medical sign to monitor ocular conditions including dry eye disease (DED), glaucoma, carotid artery stenosis, diabetic retinopathy, and vascular neuritis. The ability to measure OST in real time is desirable in point-of-care diagnostics. Here, we developed minimally invasive contact lens temperature sensors for continuous monitoring of the corneal temperature. The contact lens sensor consisted of a laser patterned commercial contact lens embedding temperature-sensitive Cholesteric Liquid Crystals (CLCs), which exhibited a fully reversible temperature-dependent color change in the visible spectrum. The contact lens allowed the corneal temperature to be mapped in four key areas, at distances of $0.0,1.0,3.0$, and $5.0 \mathrm{~mm}$ from the pupil's edge. Liquid crystals exhibited a wavelength shift from $738 \pm 4 \mathrm{~nm}$ to $474 \pm 4 \mathrm{~nm}$ upon increasing the temperature from $29.0^{\circ} \mathrm{C}$ to $40.0^{\circ} \mathrm{C}$, with a time responsivity of $490 \mathrm{~ms}$ and a negligible hysteresis. Readouts were performed using a smartphone, which output RGB triplets associated to temperature values. Contact lens sensors based on CLCs were fitted and tested on an ex vivo porcine eye and readouts were compared with infrared thermal measurements, resulting in an average difference of $0.3^{\circ} \mathrm{C}$.

Received 23rd January 2019

Accepted 5th April 2019

DOI: 10.1039/c9ra00601j

rsc.li/rsc-advances the subject (average decrease of $0.015{ }^{\circ} \mathrm{C}$ per year), ${ }^{12}$ use of eyedrops, ${ }^{\mathbf{1 , 1 3}}$ contact lens wear, ${ }^{\mathbf{1 4}}$ and tear film instability. ${ }^{8}$ OST was found not to vary between the right and left eye, and among genders. ${ }^{15}$ OST variation was targeted as a potential stimulus to blink. ${ }^{1}$ Ocular temperature was also studied in animal models to develop novel drug delivery systems. ${ }^{\mathbf{1 6}}$ The Intra-Ocular Pressure (IOP), ${ }^{17}$ corneal elasticity and related refractive properties of the eye $\mathrm{e}^{\mathbf{1 8}}$ were also demonstrated to be related to the temperature of the ocular anterior chamber. Several ocular conditions were reported to be linked to OST variations, resulting from a change in blood flow to the sclera and conjunctiva. ${ }^{19}$ OST was found to variate in cases of inflammation, such as Dry Eye Disease (DED), ${ }^{\mathbf{1 , 2 , 2 0 - 2 8}}$ glaucoma, ${ }^{29}$ diabetic retinopathy, ${ }^{30,31}$ and vascular neuritis. ${ }^{1}$ Strong positive correlations were reported between ocular temperature difference values (TDVs) and dry eye parameters, including tear meniscus height (TMH) and Schirmer's test results. ${ }^{22}$ Evaporative Dry Eye (EDE) patients were found to have a higher initial OST than those with Aqueous Deficient Dry Eye (ADDE), suggesting that OST monitoring might be an effective tool to differentiate between different DED aetiologies. ${ }^{23}$ An average increase of $0.8{ }^{\circ} \mathrm{C}$ was observed in the OST of Meibomian Gland Dysfunction (MGD) patients when compared to matched controls. ${ }^{32}$ Attempts were made in DED screening by OST measurement. ${ }^{24,25}$ The OST was found to increase as a response to peripheral vasoconstriction, suggesting OST monitoring as a promising method to evaluate ocular vasoregulation. ${ }^{33}$ An increase of $1.18^{\circ} \mathrm{C}$ in OST was found in post-corneal transplant

\footnotetext{
${ }^{a}$ Department of Chemical Engineering, Imperial College London, London, SW7 2AZ UK. E-mail: r.moreddu18@imperial.ac.uk

${ }^{b}$ School of Chemical Engineering, University of Birmingham, Birmingham, B15 2TT, UK ${ }^{c}$ Department of Mechanical Engineering, Khalifa University, Abu Dhabi 127788, United Arab Emirates

$\dagger$ Electronic supplementary information (ESI) available. See DOI: 10.1039/c9ra00601j
} 
patients undergoing an inflammation that would have leaded to a rejection of the implant. ${ }^{34}$ Differences in ocular temperature were found among phakic and pseudophakic patients when compared to matched controls. ${ }^{35}$ OST variations were also reported in dogs with keratoconjunctivitis sicca. ${ }^{36}$ Eye surface temperature showed to be correlated to stress responses in budgerigars $^{37}$ and mice. ${ }^{38}$

Corneal temperature variations may be also associated to psychiatric disorders, including schizophrenia (SCZ) and Major Depressive Disorder (MDD), due to induced alterations in thermoregulatory processes related to human homeostasis, ${ }^{15,39}$ that result in variations in core body temperature, ${ }^{40}$ as well as in superficial tissues such as the cornea. ${ }^{41}$ Corneal temperature was reported to be correlated with clinical severity of MDD and SCZ. ${ }^{39}$ Ocular infections with the parasitic apicomplexan $T$. gondii, associated to substantial increases in OST, might explain some of the perceptual distortions experienced in schizophrenia. ${ }^{42}$ Drug-free SCZ patients showed $1.55{ }^{\circ} \mathrm{C}$ higher corneal temperatures when compared to matched controls. ${ }^{43}$ Another study reported that the corneal temperature of neuroleptic-treated patients was significantly lower than that of the drug-free patients. ${ }^{44}$ OST has a high potential to be targeted as a parameter for real-time monitoring the ocular physiology. ${ }^{\mathbf{1 , 2 3 , 2 5 , 3 0 , 3 1 , 4 5}}$ Contact lenses have recently attracted particular interest to be used as wearable sensing systems for point-of-care medicine. ${ }^{\mathbf{4 6 , 4 7}}$ In particular, scleral contact lenses are ocular prosthetic devices obtained from rigid polymers with high oxygen permeability for preserving corneal metabolism. ${ }^{48}$ They rest on the sclera creating a tear fluid reservoir over the cornea. ${ }^{49}$ They are already used to treat a number of eye diseases and injuries, ${ }^{\mathbf{4 8 , 5 0}}$ including dry eye syndrome, ${ }^{51}$ keratoconus, ${ }^{48,52}$ Steven-Johnson syndrome, ${ }^{\mathbf{4 8}}$ post Laser Assisted In situ Keratomileusis (LASIK) complications, ${ }^{\mathbf{5 0 , 5 3}}$ as well as post corneal transplant complications. ${ }^{48}$ Hence, the measurement of OST on a continuous basis is highly desirable in a wide range of physiological conditions to monitor disease progression in personalized diagnostics. Here, we developed scleral contact lenses for continuous OST monitoring. Temperature-sensitive cholesteric liquid crystals based on cholesteryl oleyl carbonate (COC), cholesteryl nonanoate (CN), and cholesteryl benzoate (CB) were embedded within fluoro silicone acrylate scleral lenses (Boston XO) inside micropatterns etched in the contact lens by laser ablation. Colorimetric readouts were performed using Color Comparator and Color Name smartphone applications, ${ }^{54}$ based on the Red, Green and Blue (RGB) color model..$^{55}$

\section{Results}

Thermochromic liquid crystals (TLC) were obtained by melting cholesteryl oleyl carbonate, cholesteryl nonanoate and cholesteryl benzoate (COC/CN/CB wt\%, $0.35: 0.55: 0.10$ ). The three esters share the same molecular skeleton, bonded to a different radical (Fig. 1a). They can be melted together to obtain a liquid crystal mixture, in which each compound retains its own chemical properties, whilst forming weak inter- and intra- molecular interactions. The physical properties of the mixture, such as the temperature sensitivity range, can be modulated by varying the concentration of each compound within the mixture. Thermochromic liquid crystalline (TLC) materials have been widely reported. ${ }^{55,56}$ Their reversibility, fast response times, multiple color transitions, and high temperature accuracy $\left(0.1^{\circ} \mathrm{C}\right)$ makes them a promising alternative to electronic sensors for precision thermal mapping in wearable medicine. Chiral, rigid and rod-shaped molecules can form the cholesteric phase across a defined temperature range, exhibiting a layered structure with a gradual twisting in the molecular orientation ${ }^{55,56}$ (Fig. 1b and c). This helical arrangement results in a Bragg reflection-like mechanism where incident light normal to the molecular orientation and circularly polarized with the same sense as the helix is reflected according to the formula $\lambda=n P,{ }^{56,57}$ where $\lambda$ is the reflected wavelength, $n$ is the refractive index of the material, and $P$ is the pitch, defined as the distance between two equallyoriented layers, which variates over temperature following a non-linear dependence. This results in a more rapid shift in color at the red end of the visible spectrum compared to the blue. ${ }^{56,57}$ Upon a temperature increase during the cholesteric phase, a shortening of the helical stack is observable. The pitch decreases from $P_{1}$ to $P_{2}$, resulting in a reflection peak shift from $\lambda_{1}$ to $\lambda_{2}$, that in turns induces a color change visible at the macro scale. Fig. 1d presents a schematic of the fabrication of contact lens temperature sensors. Micropatterns are etched on scleral lenses by $\mathrm{CO}_{2}$ laser ablation. Liquid crystals are prepared and embedded in the micro-structures by UV epoxy bonding a $150 \mu \mathrm{m}$ thin, circular glass piece to the lens, around the pattern. Contact lens sensors can be rinsed in commercial saline solutions or in DI water.

\section{Characterization of the liquid crystal}

Optical characterization of liquid crystals was performed using the reflection spectroscopy setup depicted in Fig. 2a. A bifurcated optical fiber for both illumination and detection at an angle of $90^{\circ}$ was used. Standard errors were calculated from three measurements per point. Fig. $2 \mathrm{~b}$ shows the wavelength shift of the liquid crystal within the visible spectrum, from $738 \pm 4 \mathrm{~nm}$ to $474 \pm 4 \mathrm{~nm}$, upon increasing the temperature from $29.0{ }^{\circ} \mathrm{C}$ to $40.0{ }^{\circ} \mathrm{C}$ with steps of $0.5^{\circ} \mathrm{C}$. The reflection peak shift over temperature can be nicely approximated by a second order polynomial fitting $\left(R^{2}=\right.$ 0.98) that individuates a hyperbolic behavior, typical of liquid crystals (Fig. 2c). The reflection efficiency trend was measured within the same temperature range (Fig. 2d). It was found to gradually increase from $14 \pm 4 \%$ at $29.0{ }^{\circ} \mathrm{C}$ until reaching its highest value at $31.5^{\circ} \mathrm{C}$, which amounted to $46 \pm$ $2 \%$, followed by a decrease from $31.5{ }^{\circ} \mathrm{C}$ to $40.0{ }^{\circ} \mathrm{C}$. The lowest efficiency was measured at $40.0{ }^{\circ} \mathrm{C}(4 \pm 3 \%)$, where the liquid crystal exhibited a color that resembled a dark blue with the naked eye. A color can be uniquely identified by a point on the CIE 1931 chromaticity diagram, and it can be defined by a couple or a triplet of coordinates, according to different models. In this framework, the $(x, y)$ system was adopted. The 
(a)<smiles>CC(=O)[C@H]1CC[C@@]2(C)C(=CC[C@@H]3C4CCC(C(C)CCCC(C)C)[C@@]4(C)CC[C@@H]32)C1</smiles>

(i)

(b)

Solid crystal Liquid crystal Isotropic liquid

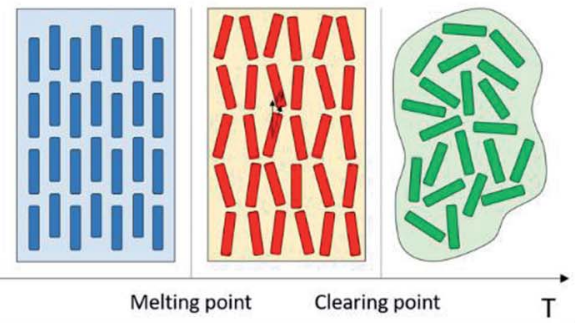<smiles>c1ccccc1</smiles>

(ii)<smiles>CCCCCC[Pb]</smiles>

(iii)

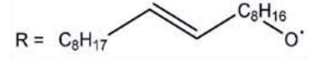

(iv) (d)

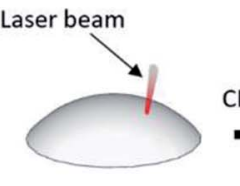

$\stackrel{\text { CLC pouring }}{\longrightarrow}$

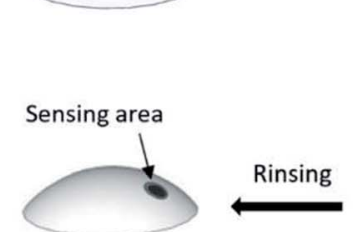

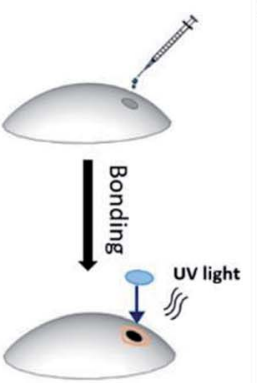

(e)

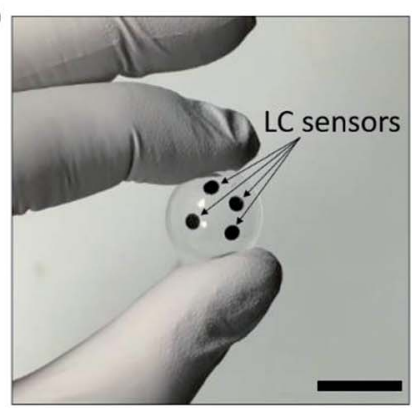

(c) Incident Reflected light

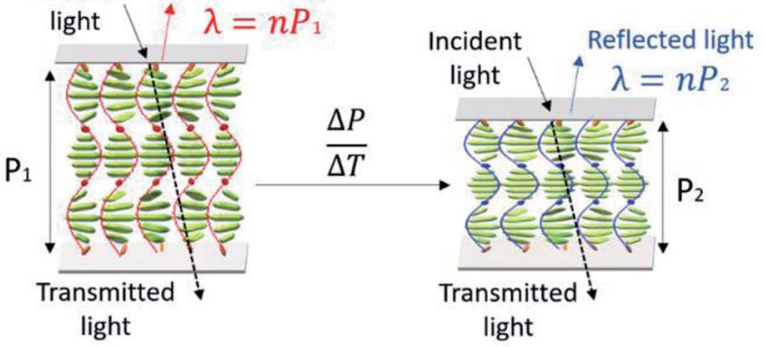

Fig. 1 Contact lens temperature sensor. (a) Chemical composition of thermochromic liquid crystals. (i) Common cholesteryl esteric skeleton, (ii) cholesteryl oleyl carbonate, (iii) cholesteryl benzoate, (iv) cholesteryl nonanoate. (b and c) Sensing mechanism of thermochromic liquid crystals: (b) temperature-triggered state change, (c) reflection peak shift over temperature in the cholesteric phase, resulting from the preferentially reflected wavelength corresponding to the distance over which a $360^{\circ}$ rotation of the field director occurs, namely the pitch, $P_{1}$ and $P_{2}$. (d) Fabrication of the contact lens sensor. (e and f) Photos of a contact lens sensor with four active areas for continuous corneal temperature mapping. Scale bars: $2.0 \mathrm{~cm}$ and $1.0 \mathrm{~cm}$.

chromaticity diagram of the liquid crystal in the temperature range $29.0-40.0^{\circ} \mathrm{C}$ with steps of $0.5^{\circ} \mathrm{C}$ is presented in Fig. 2e. The first value (1) corresponds to $29.0^{\circ} \mathrm{C}$, and the last value (23) corresponds to $40.0^{\circ} \mathrm{C}$. The $(x, y)$ coordinates in the CIE 1931 space follow a reciprocal trend (Fig. 2f). An increase in the $x$ coordinate was observed from 29.0 to $29.5{ }^{\circ} \mathrm{C}$, followed by an interval with small variations in the range $30.0-31.5^{\circ} \mathrm{C}$. A substantial decrease was measured over temperature variations in the range $32.0-34.5{ }^{\circ} \mathrm{C}$, and a minimal decrease from $35.0{ }^{\circ} \mathrm{C}$ to $38.5^{\circ} \mathrm{C}$. Small variations were observed from $39.0{ }^{\circ} \mathrm{C}$ to $40.0{ }^{\circ} \mathrm{C}$, with a peak at $39.5{ }^{\circ} \mathrm{C}$. The $y$ coordinate followed a mirrored trend to $x$, with a decrease from $29.0{ }^{\circ} \mathrm{C}$ to $31.0{ }^{\circ} \mathrm{C}$, an increase from $31.0{ }^{\circ} \mathrm{C}$ to $34.5{ }^{\circ} \mathrm{C}$, minimal variations from $34.5{ }^{\circ} \mathrm{C}$ to $36.5{ }^{\circ} \mathrm{C}$, and a gradual decrease when increasing the temperature from $36.5{ }^{\circ} \mathrm{C}$ to $40.0{ }^{\circ} \mathrm{C}$, with exception for $39.5{ }^{\circ} \mathrm{C}$, in correspondence of which a slightly larger value was measured.

\section{Smartphone readouts}

Colorimetric smartphone readouts were performed to individuate the RGB triplets identifying the colors of the liquid crystals as a response to temperature variations in the range 29.0-40.0 ${ }^{\circ} \mathrm{C}$. Color Comparator and Color Name applications installed on a smartphone were used. It is well known that RGB output triplets are dependent on the brightness and luminosity of the environment, and consequently of the material. ${ }^{55}$ Readouts were performed at ambient light levels of 20,200, and 300 lux. The phone camera was kept at normal incidence to the sensor, at a distance of $5 \mathrm{~cm}$. An illustrative example of the readout configuration is reported in Fig. 3a, where the contact lens sensors is fitted on an orbital prosthesis at ambient temperature, and the sensor displays a black color, which is detected by the smartphone app (Fig. 3b). Color Comparator used the smartphone camera to record live images of the sensor heated up at $29.0{ }^{\circ} \mathrm{C}$ to 
(a)

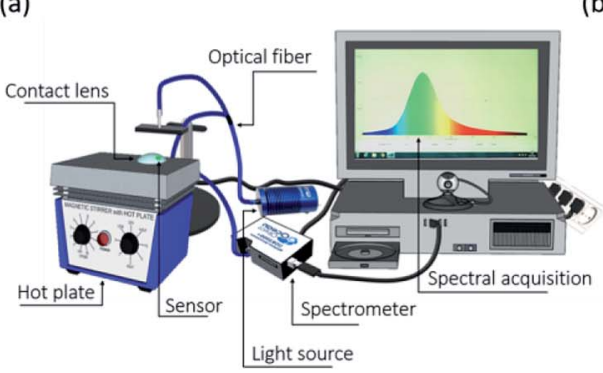

(b)

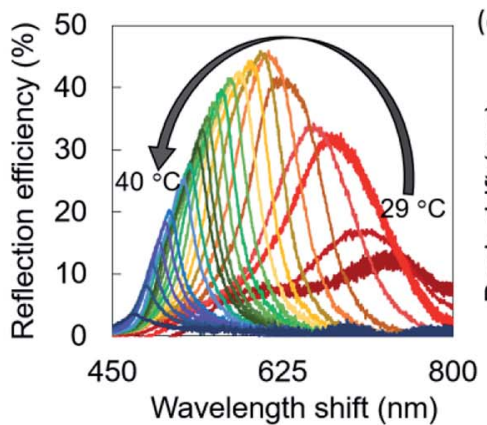

(c) 750

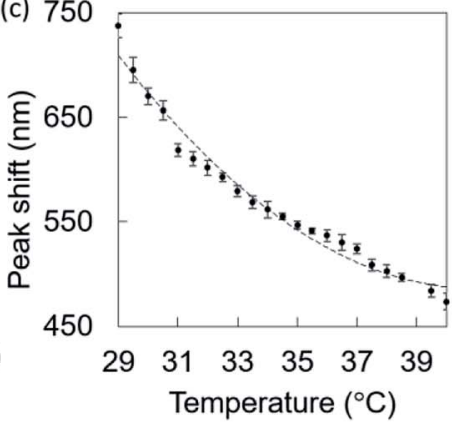

(d)

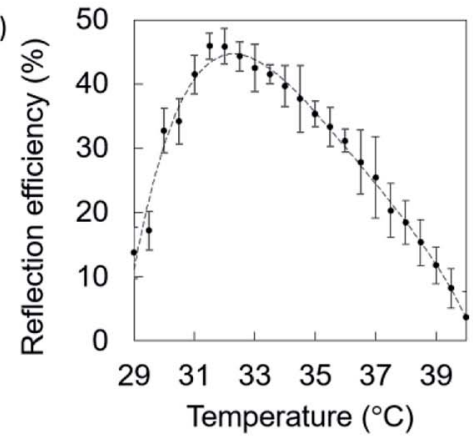

(e)

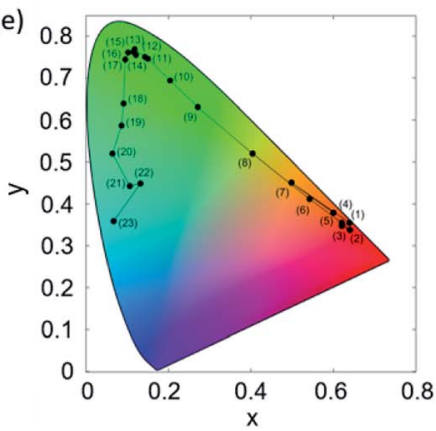

(f)

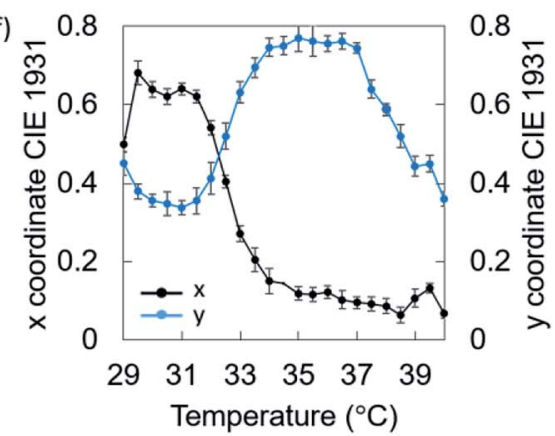

Fig. 2 Characterization of liquid crystal (COC/CN/CB wt\%, $0.35: 0.55: 0.10$ ) in the temperature range $29.0-40.0{ }^{\circ} \mathrm{C}$. (a) Reflection spectroscopy setup. (b) Wavelength shift over temperature. (c) Reflection peak shift over temperature trend. (d) Reflection efficiency over temperature trend. (e) CIE 1931 chromaticity diagram over temperature. Values from (1) to (23) correspond to reflection spectra of the liquid crystal from $29.0^{\circ} \mathrm{C}$ to $40.0^{\circ} \mathrm{C}$. (f) $\mathrm{CIE} 1931(x, y)$ coordinates over temperature trend.

$40.0{ }^{\circ} \mathrm{C}$ with steps of $0.5{ }^{\circ} \mathrm{C}$, and identified the RGB triplets associated to each temperature. Fig. $3 \mathrm{c}$ shows the RGB percentage in the range $29.0-40.0^{\circ} \mathrm{C}$ at ambient light levels of 200 lux. Note that the plot reflects a gradual shift from red to blue, where the red contribution lies between the $50 \%$ and the $80 \%$ for lower temperatures, with a further decrease. Blue contribution follows the opposite behavior, with a $25 \%$ at $29.0{ }^{\circ} \mathrm{C}$ and approaching the $60 \%$ around $39.0^{\circ} \mathrm{C}$. Green is more present for medium temperature values, lying between the $55 \%$ and the $70 \%$ for temperatures in the range $32.0-$ $36.0{ }^{\circ} \mathrm{C}$. Fig. $3 \mathrm{~d}-\mathrm{f}$ show the scatter of individual red, green and blue contributions at ambient light levels of 20,200, and 300 lux, graphically indicated with an increasing color intensity of the dots. The three scatters for each color (R, G, B) reported similar trends in the range $29.0-40.0{ }^{\circ} \mathrm{C}$, and slightly different intensities. Considering the color contributions as percentages, it is intuitive that, regardless of the intensity, a red color will exhibit a higher percentage of red, and the same concept applies to all the colors. The ambient light intensity variation is easier to appreciate when considering RGB triplets expressed in bits, where absolute red is $(255,0,0)$, absolute green is described by $(0,255,0)$ and absolute blue by $(0,0,255)$. What we can observe here is that color contributions are consistent at different light levels and they can be detected in environmental light conditions as low as 20 lux. Red peaks were reported to be at $29.0{ }^{\circ} \mathrm{C}$ and $31.0{ }^{\circ} \mathrm{C}$, where they reached the $60 \%$ and the $80 \%$ in $20-200$ and 300 lux, respectively. Green peaks were observed at
$33.5{ }^{\circ} \mathrm{C}$ and $35.5{ }^{\circ} \mathrm{C}$, with contributions of the $55 \%, 50 \%$, and $70 \%$ at 20, 200, and 300 lux, respectively. Blue peaks were found at $39.0{ }^{\circ} \mathrm{C}, 39.5{ }^{\circ} \mathrm{C}$ and $40.0{ }^{\circ} \mathrm{C}$, with contributions around the $70-80 \%$. Fig. $3 \mathrm{~g}$ shows the color of the sensor associated to different temperatures, as seen with the naked eye, captured using the smartphone camera under ambient light of 200 lux.

\section{Hysteresis}

The hysteresis of the contact lens sensor was experimentally evaluated by increasing the temperature from $33.0{ }^{\circ} \mathrm{C}$ to $38.0{ }^{\circ} \mathrm{C}$ with steps of $0.5{ }^{\circ} \mathrm{C}$, and further bringing it back to $33.0^{\circ} \mathrm{C}$, with a stabilization time of 10 minutes at each step (Fig. $3 \mathrm{~h}$ ). This range is particularly interesting to simulate temperature variations in clinical cases. ${ }^{4,6}$ Reflection peak shifts increased in all cases during the receiving mode, i.e. from $38.0{ }^{\circ} \mathrm{C}$ to $33.0{ }^{\circ} \mathrm{C}$. At $33.0^{\circ} \mathrm{C}$, the reflection peak shift varied from $655 \pm 5 \mathrm{~nm}$ to $660 \pm 3 \mathrm{~nm}$. At $34.0^{\circ} \mathrm{C}$, the reflection peak shifted from $610 \pm 8$ to $616 \pm 7$. Shifts of $573 \pm$ $8 \mathrm{~nm}$ to $579 \pm 11 \mathrm{~nm}, 553 \pm 9 \mathrm{~nm}$ to $560 \pm 5 \mathrm{~nm}$, and from 537 $\pm 11 \mathrm{~nm}$ to $546 \pm 7 \mathrm{~nm}$ were measured at $35.0^{\circ} \mathrm{C}, 36.0^{\circ} \mathrm{C}$, and $37.0{ }^{\circ} \mathrm{C}$, respectively. At $38.0{ }^{\circ} \mathrm{C}$, the reflection peak was observed at $526 \pm 6 \mathrm{~nm}$. Hysteresis was also evaluated by swinging the temperature from $32.0{ }^{\circ} \mathrm{C}$ to $33.0{ }^{\circ} \mathrm{C}$ to simulate a possible symptom of dry eye infection ${ }^{1,4,49}$ (Fig. 3i). In this case, the reflection peak shifts were found to slightly decrease in the third and fourth round when compared to the first and 
(a)

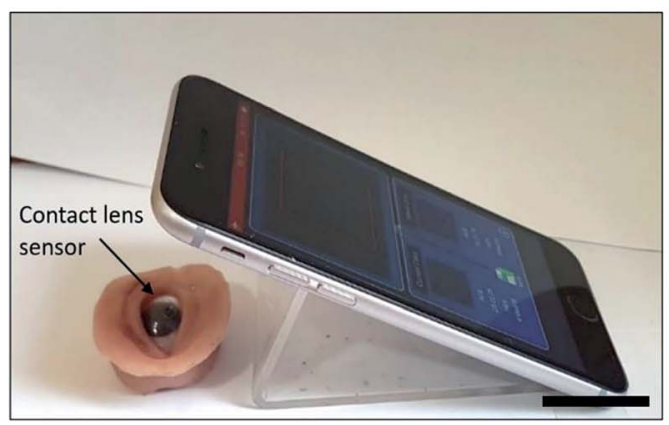

(b)

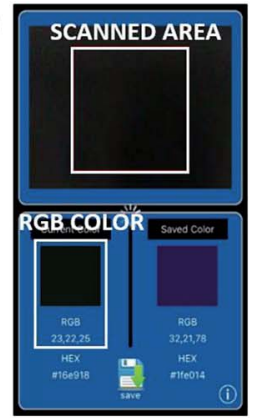

(d)

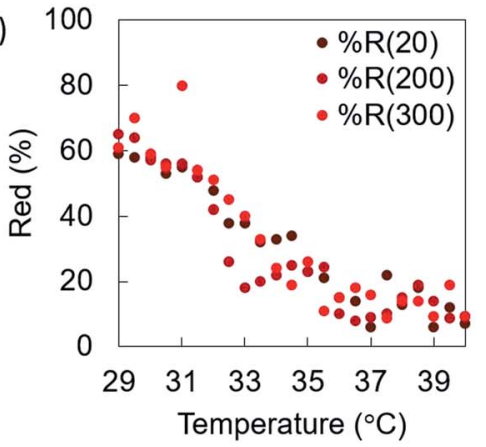

(e) 100

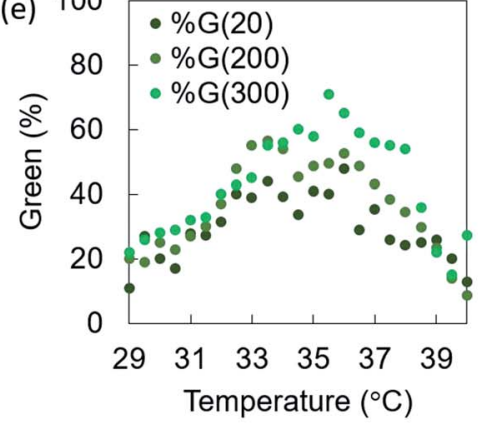

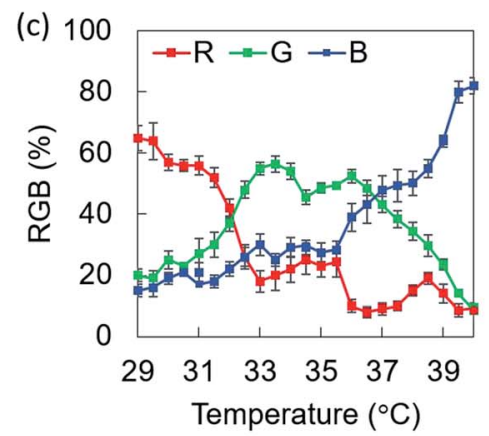

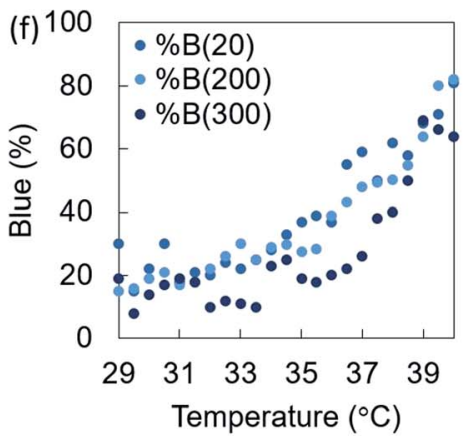

(g) $\mathrm{T}\left({ }^{\circ} \mathrm{C}\right) \quad 2929.53030 .53131 .53232 .533 \quad 33.53434 .53535 .53636 .53737 .53838 .53939 .540$

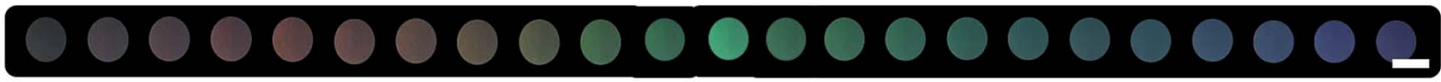

(h)

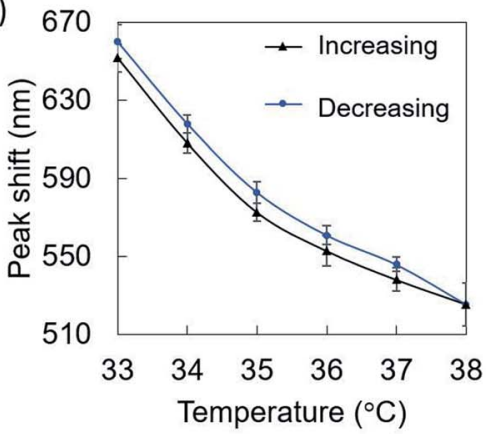

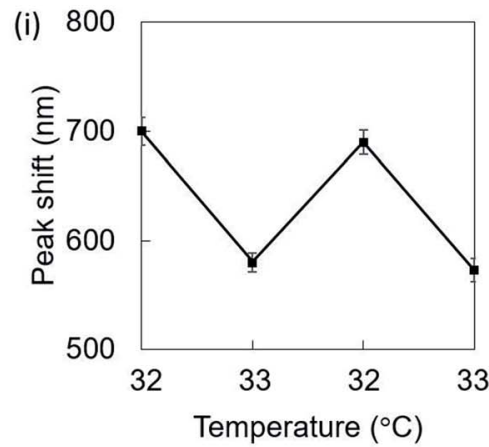

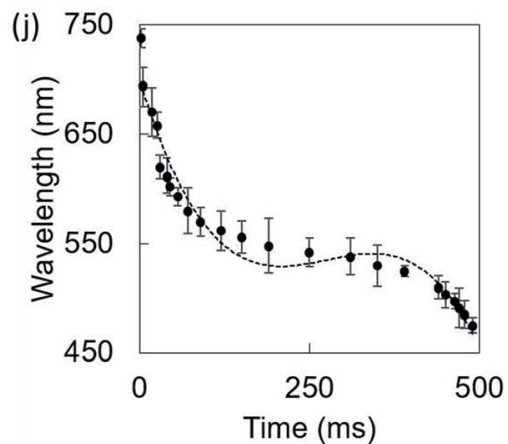

Fig. 3 response of contact lens temperature sensors. ( $a$ and b) Smartphone readout method. (a) The smartphone is placed with the camera pointing at normal incidence to the sensor, at a distance of $5 \mathrm{~cm}$. A contact lens sensor is fitted on an orbital prosthesis at ambient temperature. Scale bar: $2.5 \mathrm{~cm}$. (b) Screenshot of colorimetric readout. The black color is detected and its RGB triplet is given as an output. (c) RGB percentages in the range $29.0-40.0^{\circ} \mathrm{C}$ at ambient light 200 lux. ( $d$ and f) Individual red (d), green (e) and blue (f) contributions at ambient light levels of 20, 200 and 300 lux. (g) Color palette associated to the sensor under 200 lux in the range $29.0-40.0{ }^{\circ} \mathrm{C}$, captured with a smartphone camera. Scale bar: $2 \mathrm{~mm}$. (h) Hysteresis evaluation by swinging the temperature from $33.0^{\circ} \mathrm{C}$ to $38.0^{\circ} \mathrm{C}$ with steps of 1.0 , and back. (i) Hysteresis evaluation by swinging the temperature between $32.0^{\circ} \mathrm{C}$ and $33.0^{\circ} \mathrm{C}$. (j) Time response over temperature in the range $29.0-40.0^{\circ} \mathrm{C}$.

the second. Reflection peak shifts of $699 \pm 6 \mathrm{~nm}$ and $694 \pm$ $7 \mathrm{~nm}$ were observed at $32.0^{\circ} \mathrm{C}$; shifts of $580 \pm 5 \mathrm{~nm}$ and $573 \pm$ $4 \mathrm{~nm}$ were measured at $33.0^{\circ} \mathrm{C}$.

\section{Time response}

The time response of liquid crystals was back-evaluated based on the smartphone detection of the RGB color code associated to temperature values in the range $29.0-40.0{ }^{\circ} \mathrm{C}$ under light levels of 200 lux. The liquid crystal switched from \% RGB (65, $20,15)$, associated to a temperature of $29.0{ }^{\circ} \mathrm{C}$ to $(10,8,82)$, associated to a temperature of $40{ }^{\circ} \mathrm{C}$, in $490 \mathrm{~ms}$, with quicker response for wavelengths below $550 \mathrm{~nm}$. The time response trend approximated with a three degree polynomial curve $\left(R^{2}=\right.$ $0.96)$ is shown in Fig. 3j. A stabilization time of 1-2 seconds would be enough for ocular temperature readouts in a real-case scenario. 


\section{Characterization of liquid crystals embedded in contact lenses}

Contact lenses were fabricated to have four sensing points in key areas of the cornea, to obtain central, paracentral, peripheral, and limbal sensors. The adult cornea has a diameter of $\approx 12 \mathrm{~mm}$, and the pupil $\approx 2 \mathrm{~mm} .{ }^{1}$ The central sensor is located next to the pupil's edge, ideally at a null distance. The paracentral and peripheral sensors are located at distances of $3.0 \mathrm{~mm}$ and $5.0 \mathrm{~mm}$ from the pupil's edge, respectively. The limbal sensor is meant to lie partially on the cornea, partially on the sclera, in an area known as the limbus, where significant ocular temperature variations when compared to central corneal temperature have been reported. ${ }^{\mathbf{1}}$ A contact lens embedding four sensors was fabricated and differentially heated using a hot plate, at temperatures of $33.5{ }^{\circ} \mathrm{C}, 34.0{ }^{\circ} \mathrm{C}$, $34.5{ }^{\circ} \mathrm{C}$, and $35.0{ }^{\circ} \mathrm{C}$, leading to slightly different shades of green visible with the naked eye (Fig. 4a). Fig. 4b shows the reflection spectra recorded in the different sensors, with a shift from $542 \mathrm{~nm}$ at the limbal sensor $\left(33.5{ }^{\circ} \mathrm{C}\right)$ to $566 \mathrm{~nm}$ at the peripheral sensor $\left(35.0^{\circ} \mathrm{C}\right)$. The trends in wavelength shift and reflection efficiency observed on the contact lens sensor corresponding to different temperature are presented in Fig. 4c. Colors acquired with the smartphone app are seen as pixelated photos that may exhibit multiple color shades, and the average is automatically calculated (Fig. 4d). Three measurements per sensor were conducted and they are plotted in the CIE 1931 chromaticity diagram in Fig. 4e. Trends in wavelength shift and reflection efficiency observed on the contact lens temperature sensor at different positions are presented in Fig. 4f.

\section{Readout on an ex vivo porcine eye}

Contact lens sensors were fitted on a porcine eye and heated up at $40.0{ }^{\circ} \mathrm{C}$. Colorimetric readouts were performed on contact lenses using a smartphone and an infrared (IR) thermal camera. The results were compared. Fig. 5a shows a photo of the lens fitted on the eye with heating off and on. At ambient temperature, the sensors appear black. Shades of green and blue are visible upon switching on the heat. The sensor is imaged using a smartphone camera at normal incidence, and at a distance of $5 \mathrm{~cm}$ (Fig. 5b). The photo is uploaded in Color Name app, where colorimetric readouts are individually performed in the areas of interest (Fig. 5c). The applications output a set of RGB codes associated to the color identified in each area, and the resulting normalized color is displayed. Colorimetric results are summarized in the RGB plot of Fig. 5d. By interpolation with the RGB characteristic of contact lens sensors, previously displayed in Fig. 3c, four temperature readouts could be individuated, namely $34.5 \pm 0.4{ }^{\circ} \mathrm{C}$ at the central sensor, $36.0 \pm 0.5{ }^{\circ} \mathrm{C}$ at the paracentral sensor, $37.0 \pm 0.3{ }^{\circ} \mathrm{C}$ at the peripheral sensor, and $39.0 \pm 0.3{ }^{\circ} \mathrm{C}$ at the limbal sensor. At the same times, the eye was imaged using a smartphone-compatible IR thermal camera. Thermal images corresponding to Fig. 5a are displayed in Fig. 5e, at room temperature and under heating. At heating off, an average of the corneal temperature was extracted, with (a)

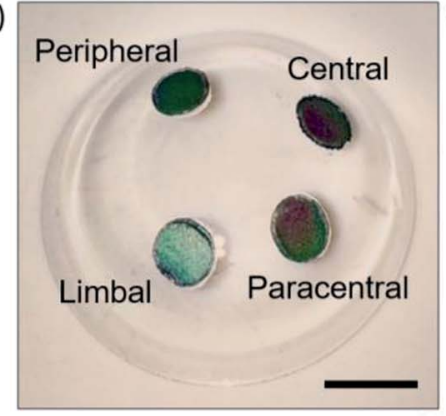

(d)

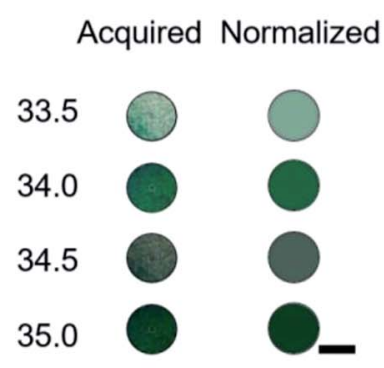

(b)

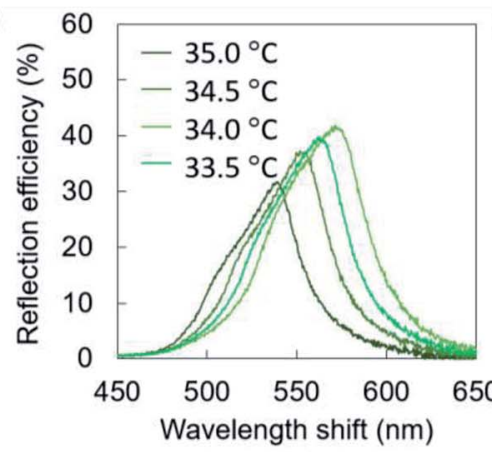

(e)

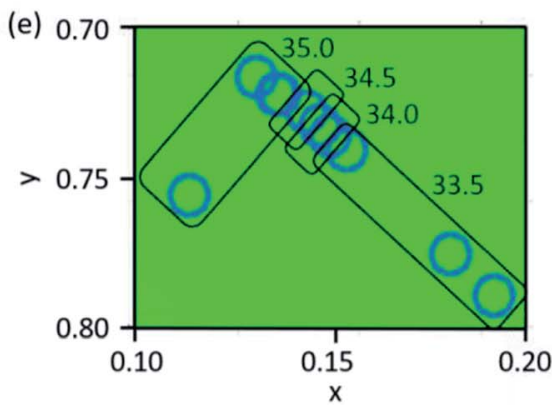

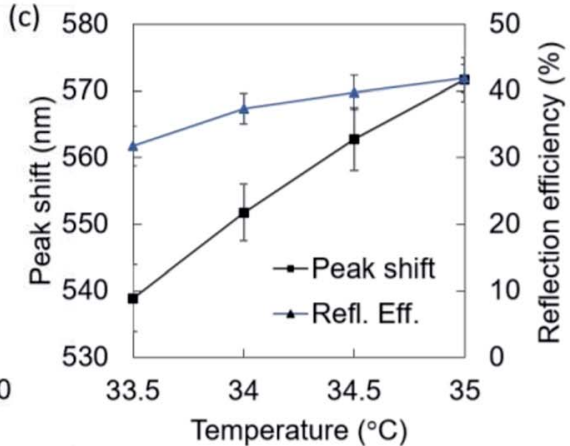

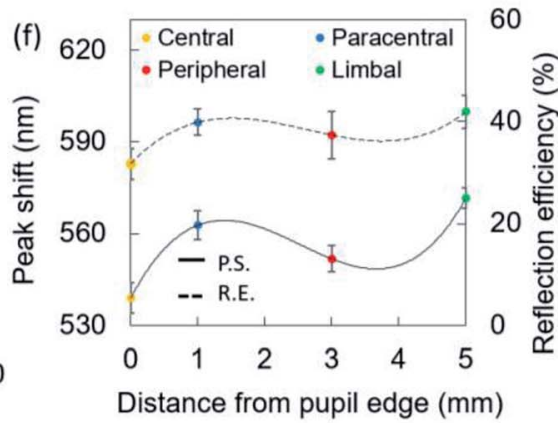

Fig. 4 Optical characterization of a contact lens temperature sensor with four active areas. (a) Photograph of a contact lens for corneal temperature mapping, differentially heated at $33.5^{\circ} \mathrm{C}, 34.0^{\circ} \mathrm{C}, 34.5^{\circ} \mathrm{C}$, and $35.0^{\circ} \mathrm{C}$. Scale bar: $1.0 \mathrm{~cm}$. (b) Reflection spectra of the four sensors. (c) Reflection peak shift and reflection efficiency trends over temperature within the contact lens. (d) Color of the four sensors, as seen when acquired and after average on the smartphone app. Scale bar: $2.0 \mathrm{~mm}$. (e) Wavelengths of the colors observed within the contact lens plotted on the CIE 1931 chromaticity diagram. (f) Reflection peak shift and reflection efficiency trends plotted over distance from pupil's edge to individuate the four sensors. 
(a)

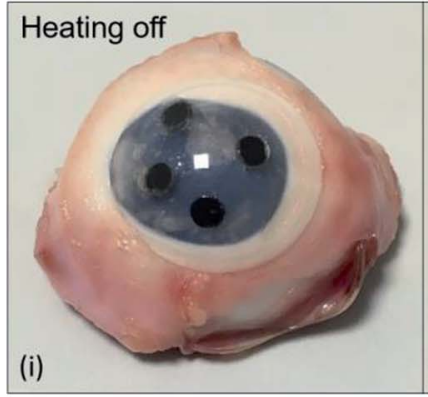

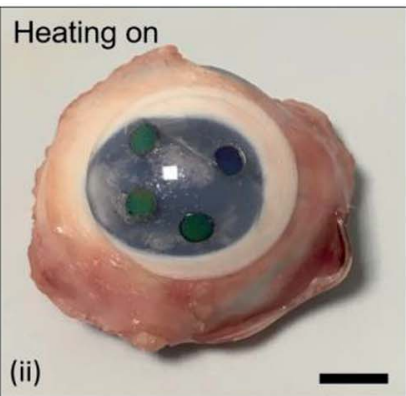

(b)

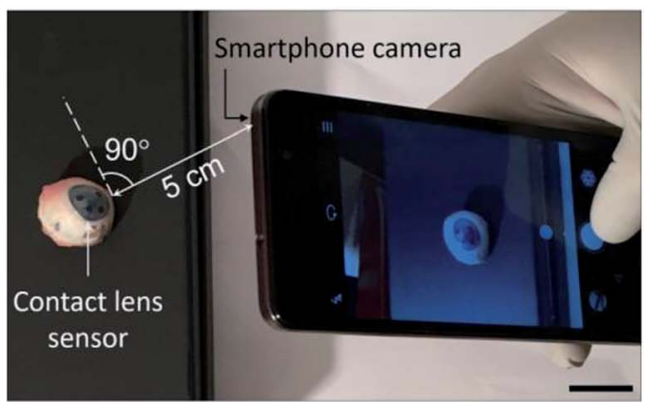

(c)

Position Acquired Normalized Readout

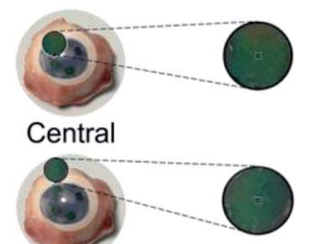

Paracentral

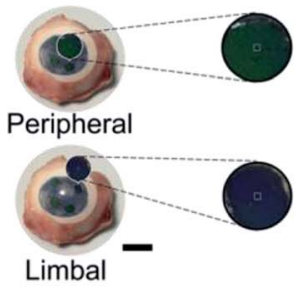

(e)

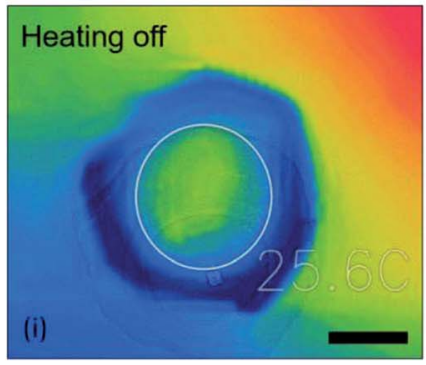

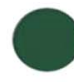
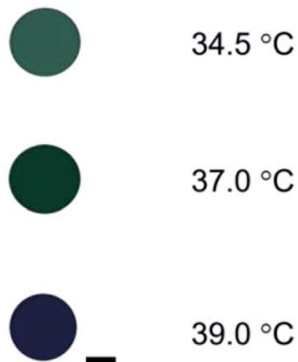

$36.0^{\circ} \mathrm{C}$

$34.5^{\circ} \mathrm{C}$

$37.0^{\circ} \mathrm{C}$

(d)
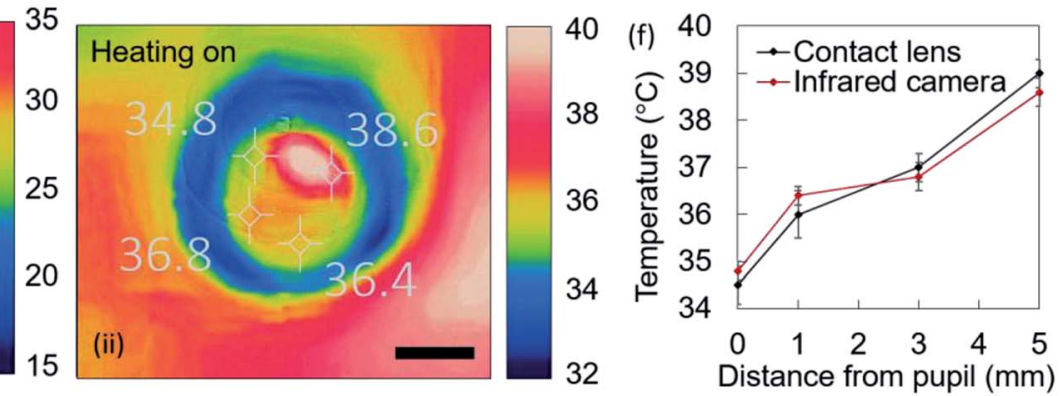

Fig. 5 Readout process tested on an ex vivo porcine eye. (a) Photograph of a contact lens sensor fitted on a porcine eye at ambient temperature (i) and under heating (ii). Scale bar: $50 \mathrm{~mm}$. (b) The sensor under heating is imaged using a smartphone at normal incidence and at a distance of $5 \mathrm{~cm}$. Scale bar: $2.0 \mathrm{~cm}$. (c) Smartphone readouts. Eyes with inset colors are shown. Scale bar of eye photos: $2.0 \mathrm{~cm}$. Scale bar of colored circles: $2.0 \mathrm{~mm}$. (d) RGB triplets associated to the temperature values measured on a contact lens sensors fitted on a porcine eye under heating. (e) IR photo of a contact lens fitted on a porcine eye at ambient temperature (i) and under heating (ii). Scale bar: $1.0 \mathrm{~cm}$. (f) Comparison of the temperature measurements using a contact lens sensor and an infrared camera.

a measured value of $25.6{ }^{\circ} \mathrm{C}$. At heating on, the four sensing points were spotted in the image, and their temperature was measured. Reported values were $34.8 \pm 0.2^{\circ} \mathrm{C} 36.4 \pm 0.2^{\circ} \mathrm{C} 36.8$ $\pm 0.3{ }^{\circ} \mathrm{C} 38.6 \pm 0.2{ }^{\circ} \mathrm{C}$. Fig. $5 \mathrm{j}$ shows the comparison between temperature values measured with the infrared camera and with contact lens sensors. An average difference of $\pm 0.3{ }^{\circ} \mathrm{C}$ was observed.

\section{Discussion}

Liquid crystals were obtained by mixing three cholesteric esters (CLC, CN, and CB), obtaining a temperature sensitivity range of $29.0-40.0{ }^{\circ} \mathrm{C}$ with steps of $0.5^{\circ} \mathrm{C}$. The same materials may be capable to discriminate among temperature intervals as small as $0.1{ }^{\circ} \mathrm{C}$ (ref. 57) when controlling the temperature at shorter steps with instruments operating with higher precision. Liquid crystals showed a decrease in the reflected wavelength for increasing temperature values. This is due to an increase in the displacement angle between the long axes of adjacent molecules in the helical stack defined in the cholesteric phase of these molecules, with a decrease in the pitch. The pitch dictates the preferentially reflected and transmitted wavebands within the material. No overlaps were observed between reflection peaks in the liquid crystal at consecutive temperature values. Overlaps were observed in the time response, suggesting that a stabilization time of $2 \mathrm{~s}$ would be 
enough for ocular temperature measurements. The reflection efficiency showed a peak at $32.0{ }^{\circ} \mathrm{C}$. The colorimetric characterization of liquid crystals was performed using smartphone apps at different light levels, and they exhibited a consistent trend. Corneal temperature values of $34^{\circ} \mathrm{C}$ and above were reported multiple times in literature, in particular as a target threshold for diagnostic purposes..$^{1,4,17,27,34}$ Other studies ${ }^{1,6,49}$ reported that temperatures in the range $32.0-33.5{ }^{\circ} \mathrm{C}$ were found across the corneal area. The hysteresis of the sensor was evaluated by temperature swing in the range $33.0^{\circ} \mathrm{C}$ to $38.0^{\circ} \mathrm{C}$, and from $32.0{ }^{\circ} \mathrm{C}$ to $33.0{ }^{\circ} \mathrm{C}$, with a stabilization time of 10 minutes at each $1.0^{\circ} \mathrm{C}$ step, showing average variations of $\approx 8 \mathrm{~nm}$ between advancing and receding measurements. The sensors took less than $500 \mathrm{~ms}$ to undergo a black-to-black shift in the full temperature range. Liquid crystals embedded in contact lenses were optically characterized, showing a consistent behavior. The readout principle was shown on an ex vivo porcine eye, where readouts were compared with infrared thermal measurements, resulting in an average difference of $0.3{ }^{\circ} \mathrm{C}$. Contact lens temperature sensors may be used to monitor dry eye disease $\left(0.5{ }^{\circ} \mathrm{C}\right.$ decrease $),{ }^{26}$ post-corneal transplant infections $\left(1{ }^{\circ} \mathrm{C}\right.$ increase $),{ }^{34}$ IOP modifications $\left(0.5^{\circ} \mathrm{C}\right.$ variations $),{ }^{17}$ as well as to monitor ocular temperature variations in individuals for research purposes, to contribute to trace the confines of healthy/unhealthy ocular temperature values, and external factors influencing the same. Scleral lenses are an attractive device for point-of-care medicine applications, being them used daily by patients suffering from a variety of diseases where corneal temperature monitoring may be desirable.

\section{Conclusions}

Contact lens sensors to monitor the ocular surface temperature were developed, using thermochromic liquid crystal esters. Cholesteryl oleyl carbonate, cholesteryl nonanoate and cholesteryl butonate were mixed ( $\mathrm{wt} \% 0.35: 0.55: 0.10)$ to obtain a temperature sensitivity range of $29.0-40.0{ }^{\circ} \mathrm{C}$ with steps of $0.5{ }^{\circ} \mathrm{C}$. A reflection peak shift from $738 \pm 4 \mathrm{~nm}$ at $29.0^{\circ} \mathrm{C}$ to 474 $\pm 4 \mathrm{~nm}$ at $40.0^{\circ} \mathrm{C}$ was observed. Temperature-sensitive contact lenses using liquid crystals as active elements have the potential to discriminate between temperature values as small as $0.1{ }^{\circ} \mathrm{C}$. Contact lens temperature sensors can be a low cost, minimallyinvasive and portable method to perform continuous corneal temperature monitoring in a range of ocular conditions. Readouts were performed using smartphones with color recognition applications, where RGB triplets identified each color, which was associated to a temperature value. Further work will be focused on improving the resolution of the contact lens sensor by analyzing the color shades while precisely controlling the applied temperature at smaller steps. A more detailed measurement of the OST at a point-of-care level can be then performed by developing a customized smartphone app that displays the temperature value to the user. Simplifying OST measurement techniques to a point of care setting could help targeting early-stage diagnosis and ocular infections monitoring.

\section{Materials and methods}

\section{Materials}

Boston XO commercial scleral lenses (diameter $=17 \mathrm{~mm}$ ) were supplied by HORUS Contact Lenses (Verona, Italy). Cholesteryl oleyl carbonate (COC), cholesteryl nonanoate (CN) and cholesteryl benzoate (CB) were purchased from Sigma Aldrich. Norland Adhesive NOA68 UV curable glue was purchased from Thorlabs.

\section{Fabrication of contact lens sensors}

Contact lens sensors were fabricated by etching microstructures within the lens and embedding CLCs mixtures. The layout of the micropatterns were previously designed on CoreDraw software. Microstructures were obtained by laser ablation, using a $\mathrm{CO}_{2}$ Rayjet laser engraver at a wavelength of $11.6 \mu \mathrm{m}$. Settings were optimized resulting in best results when using laser powers of $40-60 \%$, engraving speed $50 \%$, and standard material with a thickness of $200 \mu \mathrm{m}$. The devices were then assembled. Cholesteric liquid crystals mixtures were produced by melting COC, CN and CB (weight ratio $0.35: 0.55: 0.10$ ). The micropatterns were filled with liquid crystal. To avoid any leakage, liquid crystals were embedded by epoxy bonding a $50 \mu \mathrm{m}$ thin glass circular sheet to the contact lens, using a UV-curable glue. A tiny amount of glue was spread around the microstructures using a cotton bud. The glass top layer was then laid over the structures and cured in a UV oven for 30 minutes to achieve bonding. The contact lens was rinsed in DI water and dried at ambient temperature.

\section{Equipment}

$\mathrm{A} \mathrm{CO}_{2}$ Rayjet laser engraver was used to fabricate the structures. A customized setup was used for reflection efficiency measurements, comprising a hot plate, a bifurcated optical fiber purchased from Thorlabs, USB2000+ miniature spectrometer for VIS-NIR measurements and a tungsten halogen light source, both purchased from Ocean Optics, an infrared thermometer and a computer with a dedicated software for spectral acquisition. Imaging was performed using a Zeiss optical microscope. The optical fiber was kept firm by a probe holder, to provide irradiation and detection at normal incidence $\left(90^{\circ}\right)$. One probe was connected to a tungsten halogen light source to irradiate the lens, whilst the reflectance probe was connected to a miniaturized optical detector. The detector was connected to a computer with a dedicated software for spectral acquisition.

\section{Optical characterization of contact lens sensors}

Contact lens sensors were characterized on a hot plate using an infrared thermometer to precisely control temperature variations in a point. The thermometer was operated at normal incidence to the sensors, with a distance of $15 \mathrm{~cm}$. The sensors were exposed to temperatures in the range $29.0-40.0{ }^{\circ} \mathrm{C}$ with steps of $0.5^{\circ} \mathrm{C}$. Spectra were acquired in reflection mode and the reflection peak shift over temperature was calculated. Hysteresis was evaluated by swinging the temperature from $33.0^{\circ} \mathrm{C}$ to 
$38.0{ }^{\circ} \mathrm{C}$ and from $32.0{ }^{\circ} \mathrm{C}$ to $33.0{ }^{\circ} \mathrm{C}$. The latest measurement was meant to reproduce a dry eye symptom. ${ }^{\mathbf{1 , 4 , 6 , 4 9}}$ A stabilization time of 10 minutes was given to the hot plate before spectral acquisition. The time response of contact lens sensors was evaluated by placing the sensor on a pre-heated hot plate at $40.0{ }^{\circ} \mathrm{C}$ while continuously screen recording Color Comparator live app with a smartphone working at $60 \mathrm{fps}$. Time intervals were estimated by evaluating the time needed by the sensor to switch to specific RGB triplets, associated to temperature values in the range $29-40.0{ }^{\circ} \mathrm{C}$.

\section{Smartphone readouts}

Colors were recorded using Color Comparator ${ }^{54}$ and Color Name $^{59}$ applications, installed on an iPhone 6 s. The phone camera was placed normal to the sample at a distance of $5 \mathrm{~cm}$, under ambient light conditions of 200 lux. Light levels were measured using Lightmeter app. ${ }^{58}$ Color recognition applications identify each color with its RGB triplet. When controlling the applied temperature, each RGB triplets (or group of triplets) can be associated to a specific value. Smartphone readouts were tested on an ex vivo porcine eye. Eyes were heated on a hot plate at $40.0{ }^{\circ} \mathrm{C}$. After visual color stabilization, the readout of the sensors was performed by taking a photo and performing colorimetric analysis using Color Name app. Thermal images were acquired with a smartphone-compatible FLIR Pro camera for iOs.

\section{Conflicts of interest}

There are no conflicts to declare.

\section{Acknowledgements}

The authors thank the team of HORUS Contact Lenses (Verona, Italy) for discussions, and for providing commercial contact lenses.

\section{References}

1 C. Purslow and J. S. Wolffsohn, Ocular Surface Temperature: A Review, Eye \& Contact Lens, 2005, 31(3), 117-123.

2 J. H. Tan, et al., Infrared thermography on ocular surface temperature: a review, Infrared Phys. Technol., 2009, 52(4), 97-108.

3 S. J. Banerjee, et al., Using complex networks towards information retrieval and diagnostics in multidimensional imaging, Sci. Rep., 2015, 5, 17271.

4 J.-H. Tan, et al., Study of normal ocular thermogram using textural parameters, Infrared Phys. Technol., 2010, 53(2), 120-126.

5 J. P. Craig, et al., The role of tear physiology in ocular surface temperature, Eye, 2000, 14, 635.

6 N. Efron, G. Young and N. A. Brennan, Ocular surface temperature, Current eye research, 1989, 8(9), 901-906.

7 J. H. Tan, E. Y. K. Ng and U. R. Acharya, Evaluation of topographical variation in ocular surface temperature by functional infrared thermography, Infrared Phys. Technol., 2011, 54(6), 469-477.

8 W. Yang and L. Zhang, Association of Tear Film Stability and Corneal Surface Temperature in Pudong Patients, Curr. Eye Res., 2017, 42(5), 655-660.

9 A. A. Abusharha, E. I. Pearce and R. Fagehi, Effect of Ambient Temperature on the Human Tear Film, Eye \& Contact Lens, 2016, 42(5), 308-312.

10 J. K. Slettedal and A. Ringvold, Correlation between corneal and ambient temperature with particular focus on polar conditions, Acta Ophthalmol., 2015, 93(5), 422-426.

11 R. D. Freeman and I. Fatt, Environmental Influences on Ocular Temperature, Invest. Ophthalmol. Visual Sci., 1973, 12(8), 596-602.

12 P. B. Morgan, M. P. Soh and N. Efron, Corneal surface temperature decreases with age, Cont. Lens Anterior Eye, 1999, 22(1), 11-13.

13 S. S. Shawaf, N. Elkum and K. F. Tabbara, Effect of cold BSS vs. naphazoline $0.025 \%$ on ocular surface temperature, Eye, 2006, 20(8), 964-965.

14 C. Purslow, J. S. Wolffsohn and J. Santodomingo-Rubido, The effect of contact lens wear on dynamic ocular surface temperature, Cont. Lens Anterior Eye, 2005, 28(1), 29-36.

15 L. Tan, Z. Q. Cai and N. S. Lai, Accuracy and sensitivity of the dynamic ocular thermography and inter-subjects ocular surface temperature (OST) in Chinese young adults, Cont. Lens Anterior Eye, 2009, 32(2), 78-83.

16 F. Lorget, et al., Characterization of the $\mathrm{pH}$ and Temperature in the Rabbit, Pig, and Monkey Eye: Key Parameters for the Development of Long-Acting Delivery Ocular Strategies, Mol. Pharm., 2016, 13(9), 2891-2896.

17 C. Fabiani, et al., Relationship between Corneal Temperature and Intraocular Pressure in Healthy Individuals: A Clinical Thermographic Analysis, $J$. Ophthalmol., 2016, 2016, 3076031.

18 U. Lucia, et al., Constructal approach to bio-engineering: the ocular anterior chamber temperature, Sci. Rep., 2016, 6, 31099.

19 L. M. Parver, Temperature modulating action of choroidal blood flow, Eye, 1991, 5(2), 181-185.

20 P. B. Morgan, A. B. Tullo and N. Efron, Infrared thermography of the tear film in dry eye, Eye, 1995, 9(5), 615-618.

21 W. Mathers, Evaporation from the ocular surface, Exp. Eye Res., 2004, 78(3), 389-394.

22 T. Y. Su, et al., Correlations among ocular surface temperature difference value, the tear meniscus height, Schirmer's test and fluorescein tear film break up time, $\mathrm{Br}$. J. Ophthalmol., 2015, 99(4), 482-487.

23 S. Matteoli, et al., Ocular surface temperature in patients with evaporative and aqueous-deficient dry eyes: a thermographic approach, Physiol. Meas., 2017, 38(8), 1503.

24 L. L. Tan, S. Sanjay and P. B. Morgan, Screening for dry eye disease using infrared ocular thermography, Cont. Lens Anterior Eye, 2016, 39(6), 442-449.

25 T. Kamao, et al., Screening for dry eye with newly developed ocular surface thermographer, Am. J. Ophthalmol., 2011, 151(5), 782-791 e1. 
26 P. Versura, et al., Subjective Discomfort Symptoms Are Related to Low Corneal Temperature in Patients With Evaporative Dry Eye, Cornea, 2015, 34(9), 1079-1085.

27 L. L. Tan, S. Sanjay and P. B. Morgan, Static and Dynamic Measurement of Ocular Surface Temperature in Dry Eyes, J. Ophthalmol., 2016, 2016, 7285132.

28 I. Kovacs, et al., Abnormal activity of corneal cold thermoreceptors underlies the unpleasant sensations in dry eye disease, Pain, 2016, 157(2), 399-417.

29 F. Galassi, et al., Evaluation of ocular surface temperature and retrobulbar haemodynamics by infrared thermography and colour Doppler imaging in patients with glaucoma, $\mathrm{Br}$. J. Ophthalmol., 2007, 91(7), 878-881.

30 A. Sodi, et al., Ocular surface temperature in diabetic retinopathy: a pilot study by infrared thermography, Eur. J. Ophthalmol., 2009, 19(6), 1004-1008.

31 A. Sodi, et al., Ocular surface temperature in central retinal vein occlusion: preliminary data, Eur. J. Ophthalmol., 2007, 17(5), 755-759.

32 O. Terada, et al., Ocular surface temperature of meibomian gland dysfunction patients and the melting point of meibomian gland secretions, Nippon Ganka Gakkai Zasshi, 2004, 108(11), 690-693.

33 F. Vannetti, et al., Relationship between ocular surface temperature and peripheral vasoconstriction in healthy subjects: a thermographic study, Proc. Inst. Mech. Eng., Part $H, 2014,228(3), 297-302$.

34 M. C. Sniegowski, M. Erlanger and J. Olson, Thermal imaging of corneal transplant rejection, Int. Ophthalmol., 2018, 38(6), 2335-2339.

35 M. Sniegowski, et al., Difference in ocular surface temperature by infrared thermography in phakic and pseudophakic patients, Clin. Ophthalmol., 2015, 9, 461-466.

36 F. Biondi, et al., Infrared ocular thermography in dogs with and without keratoconjunctivitis sicca, Vet. Ophthalmol., 2015, 18(1), 28-34.

37 Y. Ikkatai and S. Watanabe, Eye surface temperature detects stress response in budgerigars (Melopsittacus undulatus), NeuroReport, 2015, 26(11), 642-646.

$38 \mathrm{~K}$. Gjendal, et al., Eye, body or tail? Thermography as a measure of stress in mice, Physiol. Behav., 2018, 196, 135-143.

39 B. Monge-Roffarello, et al., The medial preoptic nucleus as a site of the thermogenic and metabolic actions of melanotan II in male rats, Am. J. Physiol.: Regul., Integr. Comp. Physiol., 2014, 307(2), R158-R166.

40 S. Elsenga, R. H. and V. d. H., Body core temperature and depression during total sleep deprivation in depressives, Biol. Psychiatry, 1988, 24(5), 531-540.

$41 \mathrm{~J}$. J. Maller, et al., Using thermographic cameras to investigate eye temperature and clinical severity in depression, J. Biomed. Opt., 2016, 21(2), 26001.
42 E. F. Torrey and R. H. Yolken, Schizophrenia and Infections: The Eyes Have It, Schizophr. Bull., 2017, 43(2), 247-252.

$43 \mathrm{R}$. Shiloh, et al., Increased corneal temperature in drug-free male schizophrenia patients, Eur. Neuropsychopharmacol., 2003, 13(1), 49-52.

44 R. Shiloh, et al., Lower corneal temperature in neuroleptictreated vs. drug-free schizophrenia patients, Neuropsychobiology, 2003, 48(1), 1-4.

45 F. Galassi, et al., Evaluation of ocular surface temperature and retrobulbar haemodynamics by infrared thermography and colour Doppler imaging in patients with glaucoma, $\mathrm{Br}$. J. Ophthalmol., 2007, 91(7), 878-881.

46 N. M. Farandos, et al., Contact Lens Sensors in Ocular Diagnostics, Adv. Healthcare Mater., 2015, 4(6), 792-810.

47 A. K. Yetisen, et al., Wearables in Medicine, Adv. Mater., 2018, 0(0), e1706910.

48 M. M. Schornack, Scleral lenses: a literature review, Eye \& Contact Lens, 2015, 41(1), 3-11.

49 N. Efron, Contact lens practice, Elsevier, 3rd edn, 2018.

50 A. Parminder and D. S. Jacobs, Advances in scleral lenses for refractive surgery complications, Curr. Opin. Ophthalmol., 2015, 26(4), 243-248.

51 J. C. Bavinger, K. DeLoss and S. I. Mian, Scleral lens use in dry eye syndrome, Curr. Opin. Ophthalmol., 2015, 26(4), 319-324.

52 M. Frisani, M. Greco and M. Serio, Changes of ocular wave aberration in keratoconus eyes wearing mini-scleral contact lens, Cont. Lens Anterior Eye, 2018, 41(1), S50.

53 E. G. Kramer and E. L. Boshnick, Scleral lenses in the treatment of post-LASIK ectasia and superficial neovascularization of intrastromal corneal ring segments, Cont. Lens Anterior Eye, 2015, 38(4), 298-303.

54 Color Comparator - Lite (Smartphone application), downloaded September 2018 on iPhone 6s, developer: Nipakul Buttua, https://itunes.apple.com/gb/app/colorcomparator-lite/id1244696055 $\mathrm{mt}=8$.

55 T. Gevers and A. W. M. Smeulders, Color-based object recognition, Pattern Recognit., 1999, 32(3), 453-464.

56 N. Abdullah, et al., The basics and issues of Thermochromic Liquid Crystal Calibrations, Exp. Therm. Fluid Sci., 2010, 34(8), 1089-1121.

57 I. Sage, Thermochromic liquid crystals, Liq. Cryst., 2011, 38(11-12), 1551-1561.

58 Lightmeter (Smartphone application), downloaded September 2018 on iPhone 6s, https://itunes.apple.com/gb/ app/luxmeter/id1031742488? $\mathrm{mt}=8$.

59 Color Name (Smartphone application), developer: Vlad Polyanskiy, downloaded October 2018 on iPhone 6s, https://itunes.apple.com/gb/app/color-name-ar/ id $906955675 ? \mathrm{mt}=8$. 